\title{
Stab wound with transection of left vertebral artery at V3
}

\author{
Robert Bertellotti', Oluwaseye Ayoola Ogun ${ }^{1}$, Angel Mironov², Juan Antonio Asensio ${ }^{1}$ \\ ${ }^{1}$ Department of Surgery, Division of Trauma Surgery and Surgical Critical Care, Creighton University School of Medicine, Omaha, NE 68131-2137, USA. \\ ${ }^{2}$ Department of Radiology, Creighton University School of Medicine, Omaha, NE 68131-2137, USA.
}

Correspondence to: Dr. Juan Antonio Asensio, Department of Surgery, Division of Trauma Surgery and Surgical Critical Care, Creighton University School of Medicine, 601 North 30th Street, Suite 3701, Omaha, NE 68131-2137, USA. E-mail: JuanAsensio@creighton.edu

How to cite this article: Bertellotti R, Ogun OA, Mironov A, Asensio JA. Stab wound with transection of left vertebral artery at V3. Vessel Plus 2017;1:159-62.

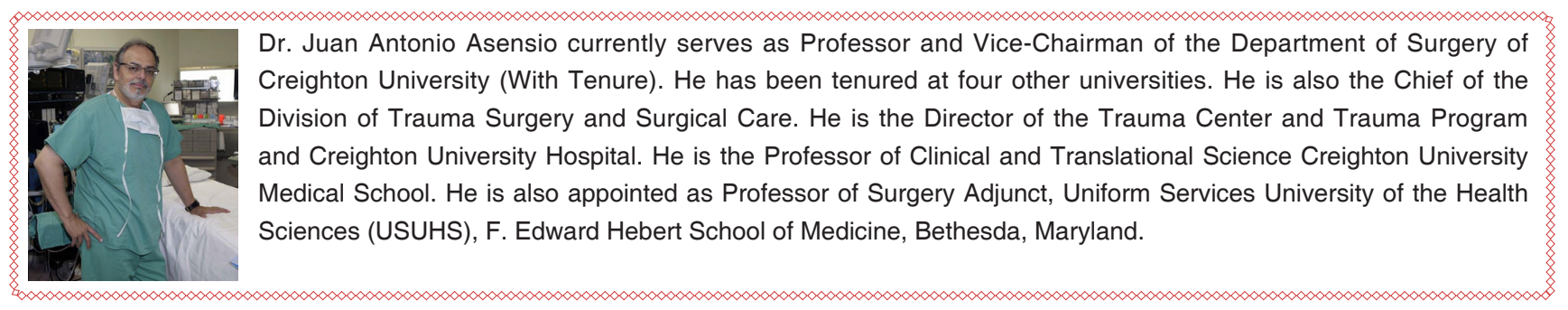

\section{Article history:}

Received: 3 Apr 2017

Accepted: 15 Jun 2017

Published: 26 Sep 2017

\section{Key words:}

Vertebral artery,

stab wound,

vertebral artery injuries

\section{INTRODUCTION}

The vertebral artery is located deep within the neck

\begin{abstract}
While vertebral artery injuries are uncommon, they can have significant morbidity if not identified and treated in a timely fashion. While the majority of vertebral artery injuries are the result of blunt injury and typically have favorable outcomes, a substantial percentage of patients with penetrating injury to the neck may also have vertebral artery injury necessitating angiographic or operative intervention. A 45-year-old male sustained a single stab wound to the apex of the posterior triangle of the neck, below the left mastoid process. At the scene, Emergency Medical Services personnel reported large blood loss and upon arrival, his initial vital signs were consistent with Class II/III hemorrhagic shock. Physical examination revealed a $9 \mathrm{~cm}$ longitudinal and deep laceration which began to bleed rapidly and profusely during his inital evaluation. The patient was intubated and rapidly transported to the operating room for exploration of the wound with direct control of the suspected vascular injury via suture ligation and application of vascular clips and to interventional radiology suite for embolization. Operative control was necessary however, immediate post-operative angiography allowed confirmation of collateral cerebral perfusion. The patient had an uneventful recovery and was evaluated in the Trauma Clinic during his 7, 14,30 and 60-day follow-up.
\end{abstract}

therefore, it is rarely injured. An epidemiology study by Hsu et al. ${ }^{[1]}$ identified 14 cases, $0.08 \%$ of vertebral artery injuries in a multi-institutional database of

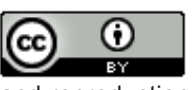

This is an open access article licensed under the terms of Creative Commons Attribution 4.0 International License (https://creativecommons.org/licenses/by/4.0/), which permits unrestricted use, distribution, and reproduction in any medium, as long as the original author is credited and the new creations are licensed under the identical terms.

For reprints contact: service@oaepublish.com

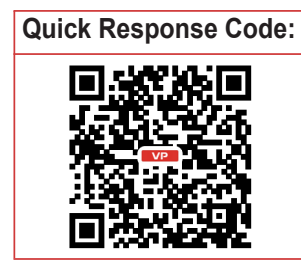


16,582 patients, a prevalence rate of 8.4 per 10,000 . When examining penetrating trauma to the neck, the incidence has been reported as between 1.0-7.4\%, with some variation attributed to mechanism (gunshot wound versus stab wound). ${ }^{[2]}$ Some believe however, that the low incidence of vertebral artery injuries previously reported is due to inefficient diagnostic tools, before the advent of routine angiography in penetrating neck trauma. ${ }^{[3,4]}$ Even with angiography, there are variations in vertebral artery anatomy that should be taken into consideration. Preoperative imaging revealed anomalous variations in $50 \%$ of patients with vertebral artery injuries, in a multicenter study. ${ }^{[1]}$ With increasing incidence of vertebral artery injury in patients with cervical spine trauma, ${ }^{[5]}$ vertebral artery injuries should be ruled out in patients presenting with neck and cervical spine trauma. ${ }^{[6]}$ Early diagnosis and intervention is critical to successful management of vertebral artery injuries. Multiple complications result from these types of injuries, and the point of entry and exit of the foreign object in penetrating injuries to the neck could predict susceptibility to injury and outcome..$^{[5,7]}$

In a prospective study by Jang et al., ${ }^{[6]}$ none of the patients experienced secondary neurologic deterioration from vertebrobasilar ischemia, similarly the patient in this case report also did not develop neurologic sequelae. Other studies have also shown that with proper management, patients experience uneventful recovery without residual effects. ${ }^{[1]}$ While neurologic deficits are rare complications of vertebral artery injury, when neurologic deficit occurs, they could be devastating and permanent. ${ }^{[1]}$ Other severe outcomes are complications of stroke, pseudoaneurysm, late-onset hemorrhage, brain stem and cerebellar infarcts, and death:; ${ }^{[1,5,8,9]}$ early diagnosis and management are therefore critical to positive outcome.

This case report aims to describe the roles of surgical procedures and interventional radiology in the successful management of emergent vertebral artery injuries.

\section{CASE REPORT}

A 45-year-old male who sustained a single stab wound at the apex of the posterior triangle of the neck below the left mastoid process was transported to the level 1 trauma center by Emergency Medical Services personnel, who reported large blood loss at the scene. Upon arrival, the patient's initial vital signs were: blood pressure (BP) 106/51 mmHg, pulse $139 \mathrm{bpm}$. Physical examination revealed a $9-\mathrm{cm}$ longitudinal deep laceration with no active bleeding and the interventional radiologist was consulted. Subsequently, the wound began to bleed rapidly and profusely. Pressure was applied. The patient was intubated; massive transfusion protocol was activated and the patient was rapidly transported to the operating room (OR).

In the OR, the wound was explored by enlarging the incision longitudinally, and interventional radiology (IR) was contacted. Ideally, the patient should have gone directly to IR suite upon his admission. Unfortunately, the patient began to bleed significantly, thus precluding this option. IR however, had already been consulted. In the OR, the goal was to obtain hemostasis through direct control of the vessel; however, the location of the injury at the skull base precluded this. Temporary control in the OR was therefore obtained.

In the OR, the sternocleidomastoid was partially transected. Bleeding was controlled from muscular arterial and venous branches. A deeper wound track in the anteromedial aspect of wound was partially explored and bleeding was controlled. The sternocleidomastoid was re-approximated and the wound was closed. Estimated blood loss (EBL) was $1,100 \mathrm{~mL}$. Total fluid replacement was $3,900 \mathrm{~mL}$ : crystalloids $2,700 \mathrm{~mL}$ and 4 units packed red blood cells (PRBCs) $1,200 \mathrm{~mL}$.

The patient was transported to the intensive care unit (ICU) pending IR arrival. Immediately upon arrival, the patient became hypotensive with a systolic BP - $55 \mathrm{mmHg}$. He bled massively. Digital control was established and he was returned to the OR.

Senior trauma surgery staff was paged signal transducer and activator of transcription to the OR. The patient was then re-explored. The longitudinal incision was extended and the sternocleidomastoid muscle was completely transected along with the splenius capitis muscle. Transverse processes of C1-C2 were palpated and the pre-vertebral fascia was entered. The vertebral artery at V3 (third portion) was noted to be partially transected extracranially; it was controlled with vascular clips. Paired vertebral veins were controlled in the same fashion. Hemorrhage was thus controlled. The accessory spinal nerve was not visualized. Bone wax was applied and the wound closed. EBL was $3,000 \mathrm{~mL}$. Total volume replacement was $6,400 \mathrm{~mL}$ : crystalloids $4,000 \mathrm{~mL}, 6$ units PRBCs $1,800 \mathrm{~mL}, 2$ units fresh frozen plasma $600 \mathrm{~mL}$.

During the second intervention, a more complete exploration identified the injury thus allowing for definitive control. 


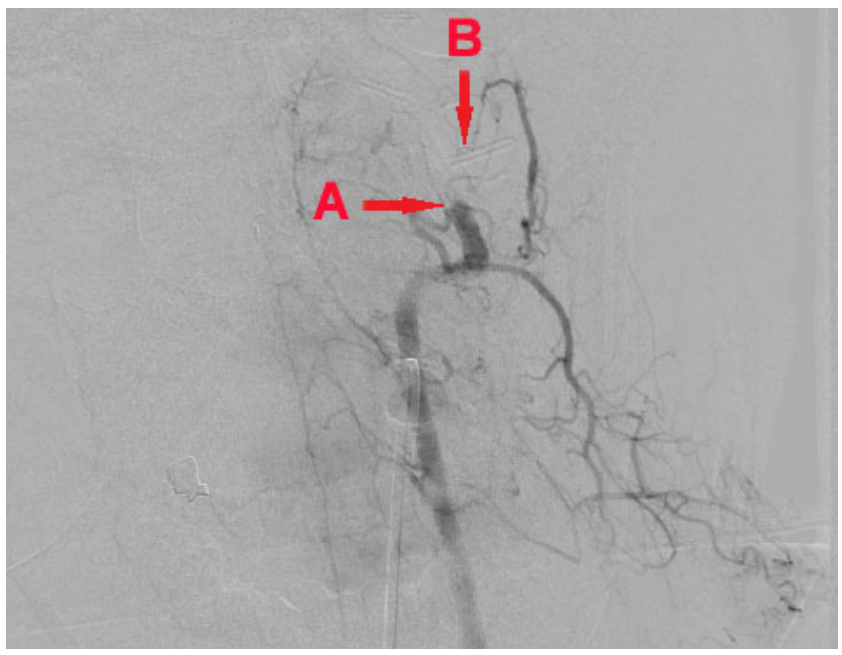

Figure 1: Vertebral artery surgically controlled prior to embolization (A) and digital subtraction shadow of surgically applied clips (B)

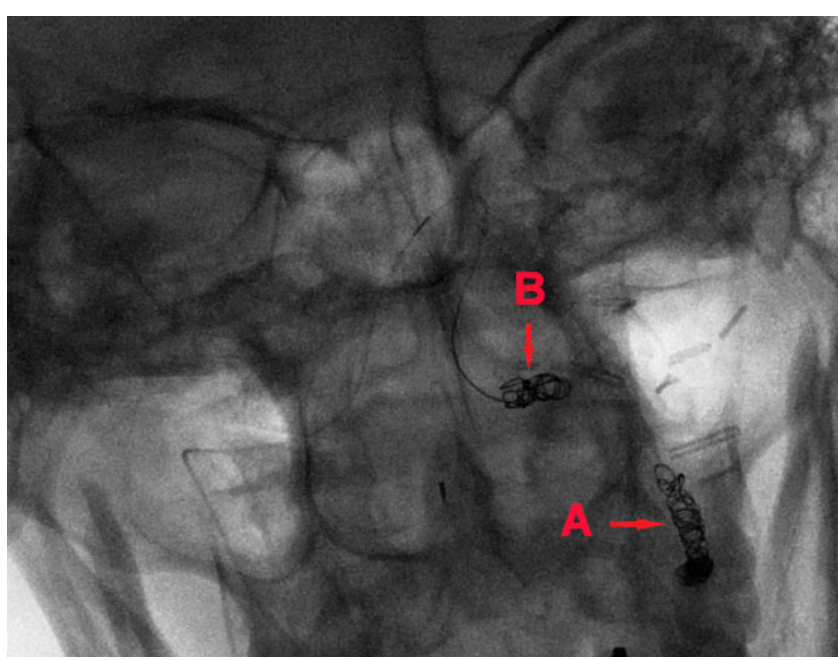

Figure 3: The micro catheter was placed in retrograde fashion in the left distal vertebral artery immediately above the extradural pseudoaneurysm during deployment. Endovascular approach obtained complete proximal $(A)$ and distal $(B)$ trapping of the extra dural left vertebral artery pseudoaneurysm above the surgical clips

Angiographic visualization of the vasculature was performed by catheter angiography, which revealed that complete acute occlusion of the left vertebral artery at the C1-C2 level was successfully achieved during the second surgical intervention [Figure 1]. The catheterization of the right vertebral artery showed normal perfusion with opacification of the very distal segment of the left vertebral artery. The late phase of angiography depicted a pattern consistent with an extra-dural pseudoaneurysm noted above the surgically placed clips.

The first step during angiography included endovascular embolization by coils of the left vertebral artery proximal to the visualized surgical occlusion. The second stage proceeded by navigation of the

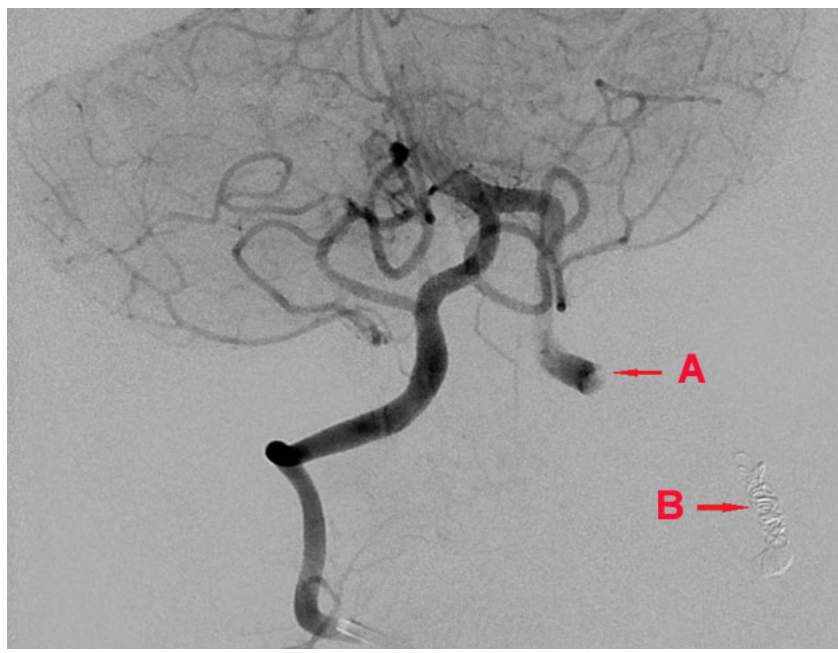

Figure 2: Right vertebral artery angiography after embolization by coils of the very proximal part of left vertebral artery (arrow showing coils, B). Retrograde opacity of the distal left vertebral artery shows persistent extravasation of contrast with an extradural pseudoaneurysm above the surgically placed clips which had controlled all bleeding operatively (A) secondary to vessel transection from the stab wound

micro-catheter through the right vertebral artery in retrograde fashion into the very distal part of the left vertebral artery with navigation of the catheter tip above the level of the surgically placed clips [Figure 2]. Embolization in this location was performed again by lumen occlusion with coils with meticulous preservation of the anterior spinal artery, which had its origin from the distal left vertebral artery. The territory of the left posterior inferior cerebellar artery was satisfactory collateralized by the left anterior inferior cerebellar artery. Thus, this endovascular approach obtained complete proximal and distal trapping of the left vertebral artery pseudoaneurysm above the surgically placed clips [Figure 3].

The patient was transferred to the ICU, extubated on the second post-operative day and transferred to the floor. He was observed for 2 days and discharged on post-operative day 4 . No problems were noted with balance when ambulating. He had an uneventful recovery. He was evaluated in the Trauma Clinic during his $7,14,30$ and 60 -day follow-up with no sequelae noted from this rare and complex vascular injury.

\section{DISCUSSION}

Penetrating vertebral artery injuries are rare and their injuries were previously missed prior to the routine use of angiography in diagnosing penetrating neck injuries.

This patient specifically, sustained a penetrating injury to the vertebral artery and had hard signs of vascular injury with profuse bleeding at the scene and upon 
examination of the wound during his initial evaluation. This is the most common presentation in penetrating vertebral artery injury at $50 \%$, while $30 \%$ present only with soft signs, and $20 \%$ with no overt signs. ${ }^{[2]}$ This frank hemorrhage was the impetus for the decision for operative exploration at the onset. This decision also corresponds to the algorithm suggested by Greer et $a{ }^{\left[{ }^{[10]}\right.}$ in their series of penetrating vertebral artery injuries managed during the military campaigns in Iraq and Afghanistan. Early operative intervention was also recommended by Reid and Weigelt ${ }^{[11]}$ in their series of 43 patients from 1976-1986; both series agree that patients without acute lifethreatening injuries can be safely evaluated without imaging studies (computed tomography angiography, digital subtraction angiography) with minimal risk to the patient.

Vertebral artery injuries though uncommon, could be fatal. Operative management is difficult and requires significant experience, in some cases, definitive control can be achieved through endovascular embolization by interventional radiology. While these approaches are described in the literature, there is little data on the multimodal therapy provided in this patient's care. Operative control was necessary however, immediate post-operative angiography allowed confirmation of collateral cerebral perfusion. The use of coil embolization in addition to ligation may also decrease the risk of post-operative morbidity such as fistula formation, re-bleeding, and pseudoaneurysm formation. Additional investigation of this approach is therefore warranted.

\section{Authors' contributions}

Study conception and design: J.A. Asensio

Analysis and interpretation of data: R. Bertellotti, O.A. Ogun, A. Mironov, J.A. Asensio

Drafting of manuscript: R. Bertellotti, O.A. Ogun

Critical revision: R. Bertellotti, O.A. Ogun, A. Mironov, J.A. Asensio

\section{Financial support and sponsorship None.}

\section{Conflicts of interest}

There are no conflicts of interest.

\section{Patient consent}

Not applicable as there is no identifying data.

\section{Ethics approval}

Not required for this type of publication in our institution.

\section{REFERENCES}

1. Hsu WK, Kannan A, Mai HT, Fehlings MG, Smith ZA, Traynelis VC, Gokaslan ZL, Hilibrand AS, Nassr A, Arnold PM, Mroz TE, Bydon M, Massicotte EM, Ray WZ, Steinmetz MP, Smith GA, Pace J, Corriveau M, Lee S, Isaacs RE, Wang JC, Lord EL, Buser Z, Riew KD. Epidemiology and outcomes of vertebral artery injury in 16,582 cervical spine surgery patients: an AOSpine North America multicenter study. Global Spine J 2017;7:S21-7.

2. Asensio JA, Trunkey DD. Current Therapy of Trauma and Surgical Critical Care. Philadelphia, PA: Mosby, Elsevier; 2008.

3. Roper PR, Guinto FC Jr, Wolma FJ. Posttraumatic vertebral artery aneurysm and arteriovenous fistula: a case report. Surgery 1984;96:556-9.

4. Golueke P, Sclafani S, Phillips T, Goldstein A, Scalea T, Duncan A. Vertebral artery injury -- diagnosis and management. J Trauma 1987;27:856-65

5. Tannoury C, Degiacomo A. Fatal vertebral artery injury in penetrating cervical spine trauma. Case Rep Neurol Med 2015;2015:571656.

6. Jang JW, Lee JK, Hur H, Seo BR, Lee JH, Kim SH. Vertebral artery injury after cervical spine trauma: a prospective study using computed tomographic angiography. Surg Neurol Int 2011;2:39.

7. Elkin DC, Harris MH. Arteriovenous aneurysm of the vertebral vessels. Ann Surg 1946;124:934-51.

8. Ohya J, Miyoshi K, Oka H, Matsudaira K, Fukushima M, Nagata K. Optimal measurement for "posterolateral protrusion" of the vertebral artery at the craniovertebral junction using computed tomography angiography. J Craniovertebr Junction Spine 2014;5:151-6.

9. Wang M. Death due to extensive cervicomedullary infarction following iatrogenic vertebral artery occlusion. Forensic Sci Med Pathol 2012;8:334-7.

10. Greer LT, Kuehn RB, Gillespie DL, White PW, Bell RS, Armonda RA, Fox CJ. Contemporary management of combat-related vertebral artery injuries. J Trauma Acute Care Surg 2013;74:818-24.

11. Reid JD, Weigelt JA. Forty-three cases of vertebral artery trauma. $J$ Trauma 1988;28:1007-12. 